\title{
Mutual effects of hexose phosphorylating enzymes and phosphorous on plant development
}

\author{
David Granot - Israel \\ Richard Amasino - USA \\ Avner Silber - Israel
}

\author{
ABSTRACT \\ Research objectives \\ 1) Analyze the combined effects of hexose phosphorylation and P level in tomato and \\ Arabidopsis plants \\ 2) Analyze the combined effects of hexose phosphorylation and P level in pho1 and pho2 \\ Arabidopsis mutants \\ 3) Clone and analyze the $\mathrm{PHO} 2$ gene \\ 4) Select Arabidopsis mutants resistant to high and low $P$ \\ 5) Analyze the Arabidopsis mutants and clone the corresponding genes \\ 6) Survey wild tomato species for growth characteristics at various P levels
}

\section{Background to the topic}

Hexose phosphorylating enzymes, the first enzymes of sugar metabolism, regulate key processes in plants such as photosynthesis, growth, senescence and vascular transport. We have previously discovered that hexose phosphorylating enzymes might regulate these processes as a function of phosphorous (P) concentration, and might accelerate acquisition of $\mathrm{P}$, one of the most limiting nutrients in the soil. These discoveries have opened new avenues to gain fundamental knowledge about the relationship between $\mathrm{P}$, sugar phosphorylation and plant development.

Since both hexose phosphorylating enzymes and P levels affect plant development, their interaction is of major importance for agriculture. Due to the acceleration of senescence caused by the combined effects of hexose phosphorylation and P concentration, traits affecting $\mathrm{P}$ uptake may have been lost in the course of cultivation in which fertilization with relatively high $\mathrm{P}(30 \mathrm{mg} / \mathrm{L})$ are commonly used. We therefore intended to survey wild tomato species for high P-acquisition at low P soil levels. Genetic resources with high P-acquisition will serve not only to generate a segregating population to map the trait and clone the gene, but will also provide a means to follow the trait in classical breeding programs. This approach could potentially be applicable for other crops as well.

\section{Major conclusions, solutions, achievements}

Our results confirm the mutual effect of hexose phosphorylating enzymes and P level on plant development. Two major aspects of this mutual effect arose. One is related to P toxicity in which HXK seems to play a major role, and the second is related to the effect of HXK on P concentration in the plant. Using tomato plants we demonstrated that high HXK activity increased leaf $\mathrm{P}$ concentration, and induced $\mathrm{P}$ toxicity when leaf $\mathrm{P}$ concentration increases above a certain high level. These results further support our prediction that the desired trait of high-P acquisition might have been lost in the course of cultivation and might exist in wild species. Indeed, in a survey of wild species we identified tomato species that acquired $\mathrm{P}$ and performed better at low $\mathrm{P}$ (in the irrigation water) compared to the cultivated Lycopersicon esculentum species.

The connection between hexose phosphorylation and P toxicity has also been shown with the P sensitive species Verticordia plumosa L . in which P toxicity is manifested by accelerated senescence (Silber et al., 2003). 
In a previous work we uncovered the phenomenon of sugar induced cell death (SICD) in yeast cells. Subsequently we showed that SICD is dependent on the rate of hexose phosphorylation as determined by Arabidopsis thaliana hexokinase. In this study we have shown that hexokinase dependent SICD has many characteristics of programmed cell death (PCD) (Granot et al., 2003). High hexokinase activity accelerates senescence (a PCD process) of tomato plants, which is further enhanced by high P. Hence, hexokinase mediated PCD might be a general phenomena.

Botrytis cinerea is a non-specific, necrotrophic pathogen that attacks many plant species, including tomato. Senescing leaves are particularly susceptible to $B$. cinerea infection and delaying leaf senescence might reduce this susceptibility. It has been suggested that $B$. cinerea's mode of action may be based on induction of precocious senescence. Using tomato plants developed in the course of the preceding BARD grant (IS 2894-97) and characterized throughout this research (Swartzberg et al., 2006), we have shown that B. cinerea indeed induces senescence and is inhibited by autoregulated production of cytokinin (Swartzberg et al., submitted).

To further determine how hexokinase mediates sugar effects we have analyzed tomato plants that express Arabidopsis HXK1 (AtHXK1) grown at different P levels in the irrigation water. We found that Arabidopsis hexokinase mediates sugar signalling in tomato plants independently of hexose phosphate (Kandel-Kfir et al., submitted).

To study which hexokinase is involved in sugar sensing we searched and identified two additional HXK genes in tomato plants (Kandel-Kfir et al., 2006). Tomato plants have two different hexose phosphorylating enzymes; hexokinases (HXKs) that can phosphorylate either glucose or fructose, and fructokinases (FRKs) that specifically phosphorylate fructose. To complete the search for genes encoding hexose phosphorylating enzymes we identified a forth fructokinase gene (FRK) (German et al., 2004). The intracellular localization of the four tomato HXK and four FRK enzymes has been determined using GFP fusion analysis in tobacco protoplasts (Kandel-Kfir et al., 2006; Hilla-Weissler et al., 2006). One of the HXK isozymes and one of the FRK isozymes are located within plastids. The other three HXK isozymes are associated with the mitochondria while the other three FRK isozymes are dispersed in the cytosol. We concluded that HXK and FRK are spatially separated in plant cytoplasm and accordingly might play different metabolic and perhaps signalling roles.

We have started to analyze the role of the various HXK and FRK genes in plant development. So far we found that $L e F R K 2$ is required for xylem development (German et al., 2003). Irrigation with different P levels had no effect on the phenotype of LeFRK2 antisense plants.

In the course of this research we developed a rapid method for the analysis of zygosity in transgenic plants (German et al., 2003).

\section{Implications, both scientific and agricultural}

$\mathrm{P}$ acquisition by plant might be increased by either enhanced expression of hexokinase, or by classic breading with wild tomato species that exhibit high $\mathrm{P}$ acquisition.

The role of specific HXK and FRK genes in P acquisition and plant development can be further examined with gene specific sense and antisense transgenic plants. 


\section{ACHIEVEMENTS}

\section{Analyze the combined effects of hexose phosphorylation and $P$ level in tomato plants}

To analyze the combined effects of hexose phosphorylation and P level on tomato plants we grew wild type tomato plants (MP-1 cv.) and its isogenic transgenic lines, HK4 and HK37, at various P levels. HK4 and HK37 lines are homozygous for Arabidopsis hexokinase 1

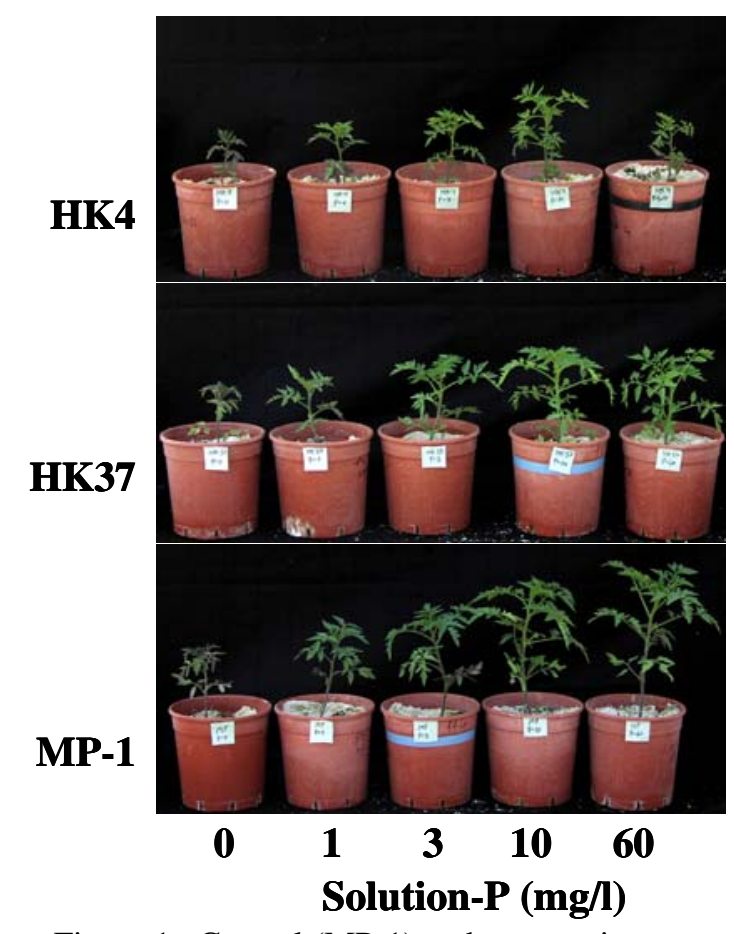

Figure 1: Control (MP-1) and transgenic tomato plants overexpressing AtHXK1, HK37 and HK4, grown at different $\mathrm{P}$ level.
(AtHXK1) that express AtHXK1 at different levels; HK4 has higher expression level of AtHXK1 compared to HK37 and accordingly exhibit higher HXK activity. As shown in Figure 1, plant growth was affected by $\mathrm{P}$ supplement. Growth of wild type plants was very poor at low $\mathrm{P}$ level and improved at increased P levels. HK37 and HK4 plants also performed better up to solution-P of $10 \mathrm{mg} / \mathrm{l}$ but at $60 \mathrm{mg} / \mathrm{l}$ the plants were slightly inhibited, while HK4 plants were further inhibited. We concluded that addition of $\mathrm{P}$ above a certain level enhanced the growth inhibition phenotype of HK plants.

To check whether solution-P indeed affected $\mathrm{P}$ content in planta we measured leaf P content in

the various lines grown at various solution-P. Leaf $\mathrm{P}$ concentration in each line increased as a function of $\mathrm{P}$ in the irrigation water (Figure 2). Furthermore, plants expressing AtHXK1 had higher leaf P concentration at each P level, directly correlated with AtHXK1 expression and activity (Figure 2). It is noteworthy that while leaf $\mathrm{P}$ concentration in wt (MP-1) plants increased up to $10 \mathrm{~g} / \mathrm{KgDW}$ as a function of solution-P, it exceeded that level in the HK plants. HK37 plants passed the $10 \mathrm{~g} / \mathrm{KgDW}$ level at about $5 \mathrm{mgP} / \mathrm{l}$ while HK4 plants passed that level at about 3mgP/l. 10g/KgDW is a high leaf $\mathrm{P}$ concentration known for many plants irrigated with "sufficient amount" of $\mathrm{P}$ (20-30 mg/l). Higher leaf P concentrations

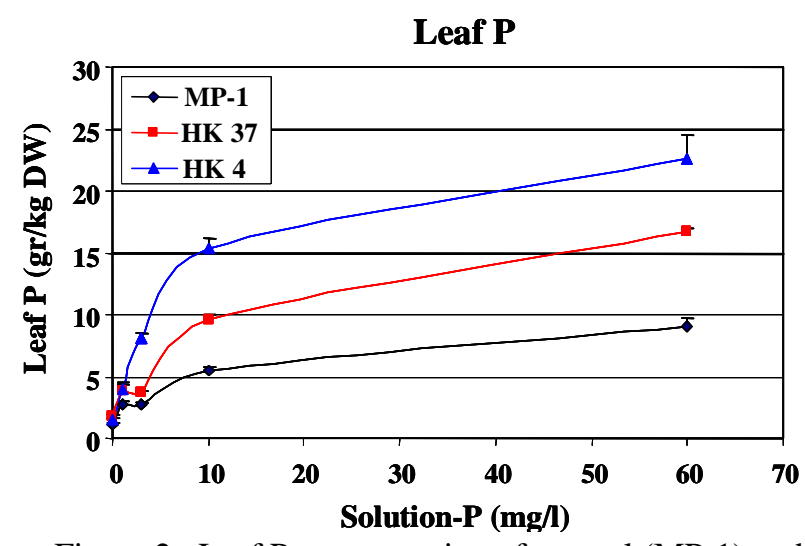

Figure 2: Leaf P concentration of control (MP-1) and transgenic tomato plants overexpressing AtHXK1, HK37 and HK4, grown at different P level. 
usually cause P- toxicity. Therefore, it was quite surprising that HK plants exceeded the $10 \mathrm{~g} / \mathrm{KgDW}$ level at relatively low solution-P. We concluded that P-toxicity like symptoms of HK plants (growth inhibition and accelerated senescence) could be the result of high leaf $\mathrm{P}$ concentration.

\section{Analyze the combined effects of hexose phosphorylation and $P$ level in pho2}

\section{Arabidopsis mutants}

pho2 mutants grown at low (1mg/l) and high (30mg/l) P had high leaf P concentration compared to w.t. plants and exhibit P toxicity symptoms (Figure 3A\&B). To check the possible correlation of pho2 phenotype with HXK activity we measured hexose phosphate within pho2 leaves grown at low and high $\mathrm{P}$. In both cases pho2 mutants had higher Glu-6P level compared to w.t. plants, though at 30 mg/l P Glu-6P was two times that of $1 \mathrm{mg} / \mathrm{l} \mathrm{P}$ (Figure 3C). These results suggest that $\mathrm{P}$ and HXK may play a role in Arabidopsis plants similar to that observed in w.t. and HK tomato plants.

\section{Survey wild tomato species for growth}

\section{characteristics at various $P$ levels}

The following wild tomato species were grown at low (1mg/l) and high (10 mg/l) P. The numbers at the end of each species represent independent accessions.

1. Lycopersicon chmielewskii 1- LA 3648

2. Lycopersicon chmielewskii 2- LA 1306

3. Lycopersicon chmielewskii 3- LA 2695

4. Lycopersicon hirsutum 1- LA 2174

5. Lycopersicon hirsutum 2- LA 2098

6. Lycopersicon hirsutum 3- LA 407

7. Lycopersicon hirsutum 4- LA 1366

8. Lycopersicon hirsutum 5- LA 2204

9. Lycopersicon hirsutum 6- LA 2650

10. Lycopersicon parviflorum 1- LA 3660

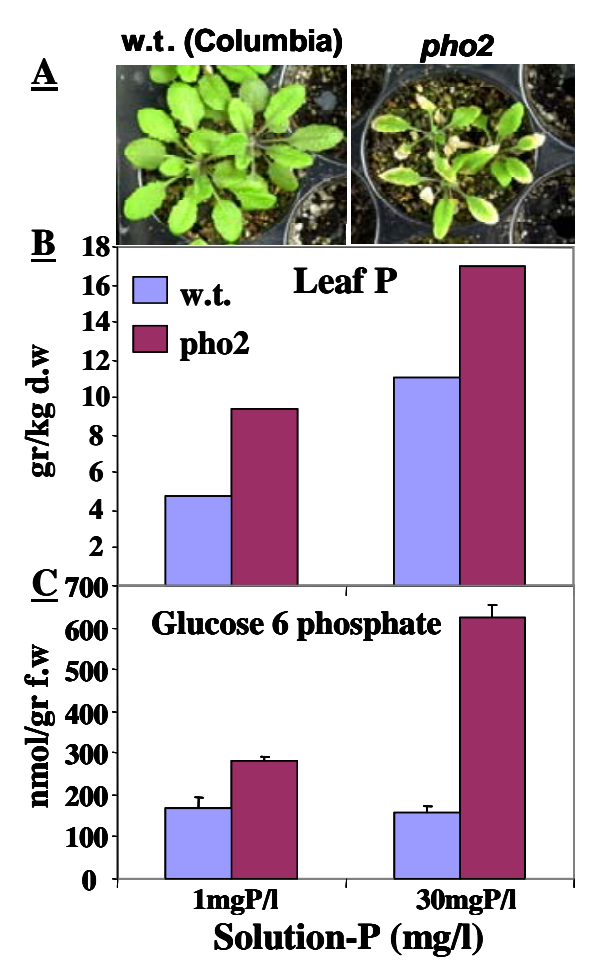

Figure 3: Effect of solution-P on Arabidopsis plants. 
11. Lycopersicon parviflorum 2- LA 1322

12. Lycopersicon pennellii- LA 716

13. Lycopersicon peruvianum 1- LA 444

14. Lycopersicon peruvianum 2- LA 1027

15. Lycopersicon peruvianum 3- LA 1677

Dry weight and leaf-P have been measured. The dry weight and leaf P of 4 of the $6 \mathrm{~L}$.

hirsutum accessions grown at low $\mathrm{P}(1 \mathrm{mg} / \mathrm{l})$ was significantly higher compared with that of control (MP-1) plants (Figure 4A\&B). It is likely therefore that these L. hirsutum accessions have the ability to accumulate $\mathrm{P}$ under $\mathrm{P}$ starvation conditions which is a desired trait for cultivated species.

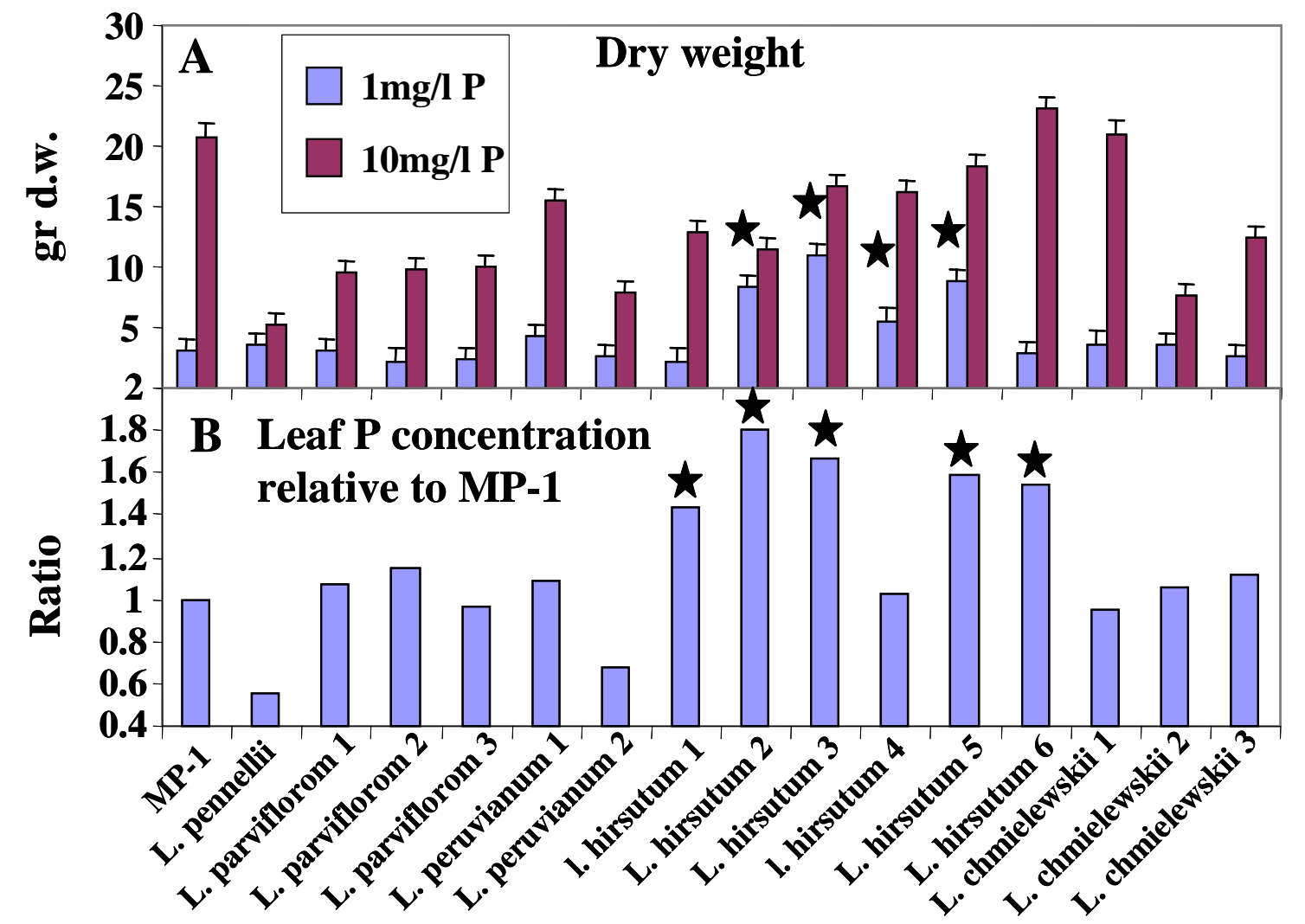

Figure 4: Dry Weight and relativve leaf P concentration of w.t. (MP-1) and the wild tomato species.

A. Dry weight measured with plants grown at $1 \mathrm{mg} / \mathrm{l}$ and $10 \mathrm{mg} / \mathrm{l} \mathrm{P}$.

B. P concentration of plants grown at $1 \mathrm{mg} / \mathrm{l}$ P relative to that obtained with the control MP-1 plants.

\section{Description of the cooperation}

\section{Description Cooperation:}

The US group provided the Israeli group with the mutagenized Arabidopsis seeds that were used for the selection experiment and guided the Israeli group how to proceed with the selected mutants. Two big selection screens were done with seeds of approx. 100,000 M2 plants obtained from EMS mutagenized Arabidopsis seeds. The selections were done in both 
low $\mathrm{P}$ (no addition of $\mathrm{P}$ to the low level of $\mathrm{P}$ already existing in drinking water) and high $\mathrm{P}$ (100mg/l). Only few mutants able to develop better than the control plants were obtained on low P. About 15 mutants that were able to survive on high $\mathrm{P}$ were also isolated. The Israeli group sent to the US group the potential mutants. However, none of the mutant lines exhibited reliable and tractable phenotypes when grown at either high or low P. Clear detectable phenotype is required for the cloning process, and therefore we could not proceed with gene cloning of the corresponding mutants.

To clone the $\mathrm{PHO} 2$ gene we have tried to map the pho2 mutation using $2 \mathrm{BAC}$ clones obtained from Manny Delhaize who originally mapped pho2 to chromosome 2. These BAC clones were designed to allow complementation following transformation with the entire BACs. The Israeli group sent to the US group the BAC clones obtained from Manny Delhaize. These BACs were analyzed by the US group and were found to be inappropriate. All the efforts to correlate between the BAC clones and the corresponding sequences on chromosome 2 failed. Meanwhile, a researcher at Mark Stitt lab (Germany) got interested in pho2 mutant and cloned the gene. To avoid futile competition, we did not pursue the cloning of pho2.

\section{Agricultural and/or economic impacts of the research findings}

A major agricultural impact of this research is the potential use of wild species of major crops (such as maize and wheat) to enhance $\mathrm{P}$ acquisition through classic breeding.

\section{List of Publications}

1. Silber A., Ben-Jaacov J., Ackerman A., Bar-Tal A., Levkovitch I., Matsevitz-Yosef T., Swartzberg D., Riov J. and Granot D. (2002). Interrelationship between phosphorus toxicity and sugar metabolism in Verticordia plumosa L. Plant and Soil 245: 249-260

2. German M., Dai N., Matsevitz T., Hanael R., Petreikov M., Bernstein N., Ioffe M., Shahak Y., Schaffer A.A. and Granot D. (2003). Suppression of fructokinase encoded by LeFRK2 in tomato stem inhibits growth and causes wilting of young leaves. Plant Journal 34:837-846

3. Granot D., Levine A. and Dor-Hefetz E. (2003). Sugar-induced apoptosis in yeast cells. FEMS Yeast Research 4:7-13

4. German M.A., Kandel-Kfir M, Swartzberg D., Matsevitz T. and Granot D. (2003). A rapid method for the analysis of zygosity in transgenic plants. Plant Science 164:183-187 
5. German M.A., Asher I., Petreikov M., Dai N., Schaffer A. A. and Granot D. (2004). Cloning, expression and characterization of LeFRK3, the fourth tomato (Lycopersicon esculentum Mill.) gene encoding fructokinase. Plant Science 166:285-291 6. Kandel-Kfir M., Damari- Weissler H., German M. A., Gidoni D., Mett A., Belausov E., Petreikov M., Adir N. and D. Granot (2006). Two newly identified membrane- associated and plastidic tomato HXKs: characteristics, predicted structure and intracellular localization. Planta (in press)

7. Swartzberg D., Dai N., Gan S., Amasino R. and Granot D. (2006). Effects of cytokinin production under two SAG promoters on senescence and development of tomato plants. Plant Biology (in press)

8. Damari-Weissler H., Kandel- Kfir M., Gidoni D., Mett A., Belausov E., Granot D. (2006). Evidence for intracellular spatial separation of hexokinases and fructokinases in tomato plants. Planta (in press) 


\section{APPENDIX}

\section{Published papers}

1. Silber A., Ben-Jaacov J., Ackerman A., Bar-Tal A., Levkovitch I., Matsevitz-Yosef T., Swartzberg D., Riov J. and Granot D. (2002). Interrelationship between phosphorus toxicity and sugar metabolism in Verticordia plumosa L. Plant and Soil 245: 249-260

2. German M., Dai N., Matsevitz T., Hanael R., Petreikov M., Bernstein N., Ioffe M., Shahak Y., Schaffer A.A. and Granot D. (2003). Suppression of fructokinase encoded by LeFRK2 in tomato stem inhibits growth and causes wilting of young leaves. Plant Journal 34:837-846 3. Granot D., Levine A. and Dor-Hefetz E. (2003). Sugar-induced apoptosis in yeast cells. FEMS Yeast Research 4:7-13

4. German M.A., Kandel-Kfir M, Swartzberg D., Matsevitz T. and Granot D. (2003). A rapid method for the analysis of zygosity in transgenic plants. Plant Science 164:183-187

5. German M.A., Asher I., Petreikov M., Dai N., Schaffer A. A. and Granot D. (2004). Cloning, expression and characterization of LeFRK3, the fourth tomato (Lycopersicon esculentum Mill.) gene encoding fructokinase. Plant Science 166:285-291

\section{'in press' and 'submitted' papers}

6. Kandel-Kfir M., Damari- Weissler H., German M. A., Gidoni D., Mett A., Belausov E., Petreikov M., Adir N. and D. Granot (2006). Two newly identified membrane- associated and plastidic tomato HXKs: characteristics, predicted structure and intracellular localization. Planta (in press)

7. Swartzberg D., Dai N., Gan S., Amasino R. and Granot D. (2006). Effects of cytokinin production under two SAG promoters on senescence and development of tomato plants. Plant Biology (in press)

8. Damari-Weissler H., Kandel- Kfir M., Gidoni D., Mett A., Belausov E., Granot D. (2006). Evidence for intracellular spatial separation of hexokinases and fructokinases in tomato plants. Planta (in press)

9. Kandel-Kfir M., Dai D., Cohen-Peer R., Matsevitz T., Swartzberg D., Silber A. and Granot

D. Arabidopsis hexokinase mediates sugar signaling in tomato plants independently of hexose phosphate. (submitted)

10. Swartzbeg D., Kirshner B., Elad Y. and Granot D. Botyrtis cinerea induces senescence and is inhibited by autoregulated production of cytokinin (submitted)

11. Granot D. Role of tomato hexose kinases. (submitted) 